\title{
REDUCING ENERGY CONSUMPTION IN SINGLE-HOP AND MULTI-HOP TOPOLOGIES OF ROAD LIGHTING COMMUNICATION NETWORK
}

\author{
Musa Cibuk \\ Department of Computer Engineering, Bitlis Eren University, Turkey \\ E-mail:mcibuk@beu.edu.tr
}

\begin{abstract}
This study aims to make the wireless sensor network based on a linear topology required in road lighting energy-efficient using the proposed new methods. Because the physical installation of road lighting systems will result in costliness and time-labour loss, the mentioned scenarios were created and analysed in a simulation design. Two new methods were proposed to organize the lighting system more quickly and to increase the speed performance of sensors that join the network and carrying the luminaire data. This is the proxy-based network connection method and a new time-division method for the nodes' common channel access. Energy consumption scenarios for lighting systems with 50 , 100, 150, and 200 luminaires were analysed comparatively during data exchange using wireless sensor networks. Accordingly, the classical method and the proposed novel method were evaluated for the single- and multi-hop scenarios. In the communication between luminaires, the proposed new method for a single-hop scenario was at least $80 \%$ more efficient than the classical method in terms of total energy consumption. In linear topology lighting systems for the same scenario, if the classical method is compared with the proposed new method for 3-hop structures, $58 \%$ efficiency of total energy consumption is achieved.
\end{abstract}

Keywords: intelligent lighting, wireless sensor network, energy efficiency, linear lighting

\section{INTRODUCTION}

In the linear wireless sensor network (WSN), nodes are ordered one after the other. The data is transmitted from the source node to the coordinator via other nodes. As the number of nodes increases, the end-to-end delay of packets and the data traffic of nodes near the coordinator increases. In addition, if the number of nodes in linear topology increases, the probability of collision and jamming increases. Protocols developed for ideal linear topologies need characteristics such as being delay-sensitive, having no connection problems, and error-free transmission of data to the centre.

With energy consumption, the rate of lighting in total energy consumption increases day by day. Intelligent lighting systems are needed to provide a sustainable and uninterrupted lighting service [15]. Wireless networks are frequently used for intelligent lighting in automation of remote monitoring and remote control systems [6-9].

Nodes are heavily used in many WSN applications. The increasing number of nodes is preventing WSN from organizing quickly. Connection patterns and sequences of nodes (topology) have a direct effect on the performance and life span of the network $[10,11]$. Topology selection is important for self-organization of the network, reducing collisions on communication lines, reducing jams and interferences, saving energy and extending network lifetime $[12,13]$. However, there is no choice of topology in linear road lighting. For linear road lighting, it is mandatory to use WSNs with linear topology 


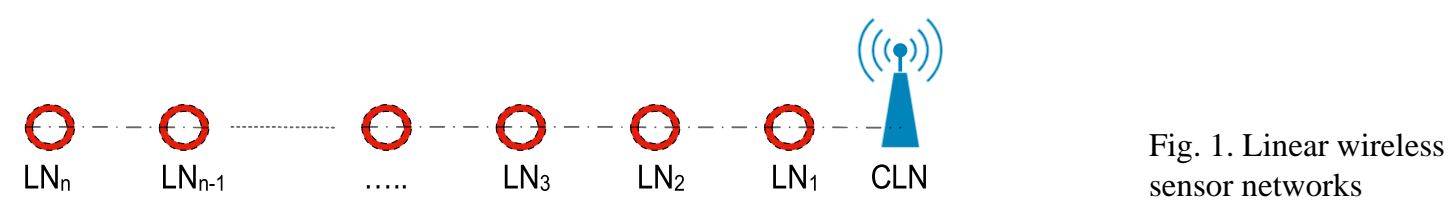

Coordinator Lighting Node (CLN)

Lighting Node (LN)

[14-16]. Therefore, in road lighting with linear topology, we need to prevent data conflicts and jams of WSN during communication and reduce interference. We should be especially careful to use energy savings when performing these operations $[8,17]$.

In this study, an efficient novel method was proposed to minimize the energy expended by linear topology based WSN in road lighting. Each lighting node (LN) in this work represents a luminaire. The transformer pole is also called the coordinator lighting node (CLN).

The remote monitoring and control system that is subject of this study records various parameters. They are the luminance level of the LNs belonging to the luminaire, the decrease in luminous flux compared to the first day's luminance level, fog in the air, humidity, air temperature regardless the luminaire is turned on or not, and the angular position of the luminaire compared to the location, etc. In this way, the information coming from the luminaires will be collected and the information about the maintenance factor of the road lighting system will be reached by automation $[8,16,18-20]$.

\section{APPLYING WIRELESS SENSOR NETWORKS TO LIGHTING}

The two basic elements that constitute the network in linear WSNs for illumination purposes are CLN and LN. CLN organizes the network and manages the LNs, enabling data communication. If the number of luminaires is small and there is no linear topology, then the communication of LNs is usually achieved by single-hop communication. If the lumi- naire topology is linear, then multi-hop communication is required. LNs that do not have direct access to CLN reach it using other LNs due to the long-distance [8, 16, 18-20]. Fig. 1 shows an example of a linear WSN.

Lighting with a linear topology is performed along the line of the road, as shown in Fig. 1. This sequence is the most widely used topology in road and tunnel lighting. [4, 6, 21, 22].

In linear topology, the LNs transmit the information of the previous LN to the CLN. LNs can be transferred with single or multi hops. As the distance between the luminaires increases, the amount of energy consumed by the nodes per packet increases. In addition, the number of hops directly affects the end-to-end delay of packets. As the number of luminaires increases, the end-to-end delay of information packets increases. Data exchange traffic on LNs close to the CLN increases in direct proportion to the size of the network. As in this study, the collision and the jam is increased with increasing number of luminaires in lighting systems with linear topology. Therefore, since the road lighting created for the lighting system must have a linear topology, the developed protocols must be delay-sensitive, have no connection problems, and have characteristics such as transmitting the data to the centre without errors [8, 23-25]. In WSNs based on linear topology, the delivery of information packets between the luminaires themselves (LN) and to the CLN is represented in Fig. 2 as 1-hop, 2-hop, and 3-hop.

When choosing the optimum conditions targeted during data exchange in WSNs, you can rely on the following parameters:

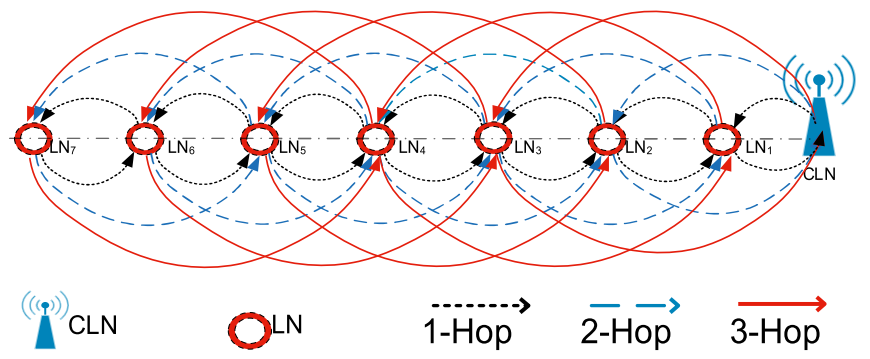

Fig. 2. 1-hop, 2-hop, and 3-hop linear WSN structure 


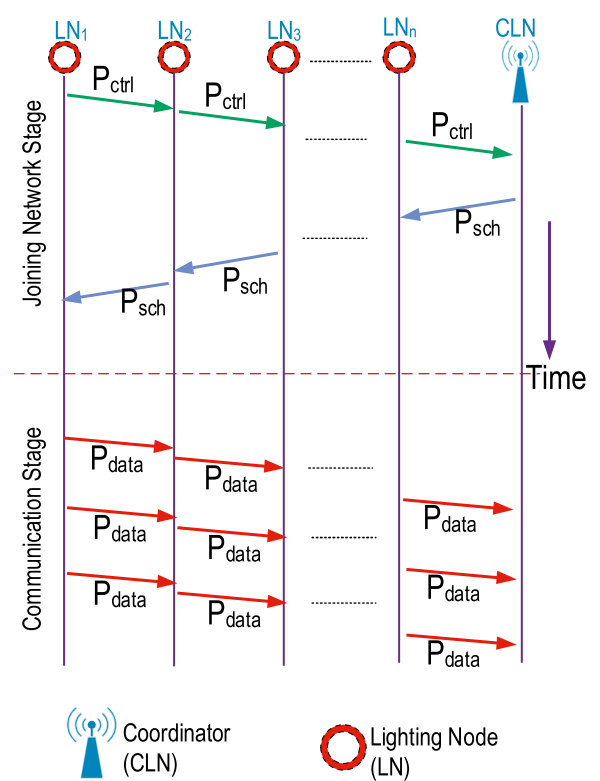

Fig. 3. The classic approach to the network joining in WSN

- low energy consumption;

- non-conflicting information packets;

- joining all nodes to the network;

- not being late in joining the network;

- not breaking out, and if the whole system is disabled for any reason;

- data exchange must be provided in a short time when the system is restarted $[8,25,26]$.

In a single-hop connection model, lighting nodes (LN) communicate directly with the CLN. In the multi-hop connection model, CLN communicates with LNs that are out of coverage zone through LNs that are in the coverage zone. Therefore, in multihop WSNs, LNs have different energy consumption. That is why the amount of data exchange is different for each LN. Since LNs close to CLN serve as routers for more remote LNs, the amount of data exchange is significantly higher than the rest. This causes LNs close to CLN to consume more energy. Therefore, when the energy of LNs close to CLN is rapidly depleted, the communication with WSN is interrupted. In remote LNs connected to this LN, even if their energy is not exhausted, they are disconnected from the network. Therefore, the data exchange is interrupted. In such cases, the topologies using the energy efficiently should be preferred to make WSN implementation optimal. However, the use of linear topology is mandatory in road lighting. Therefore, it is necessary to develop solutions to the problems of WSN in linear road lighting systems [8].

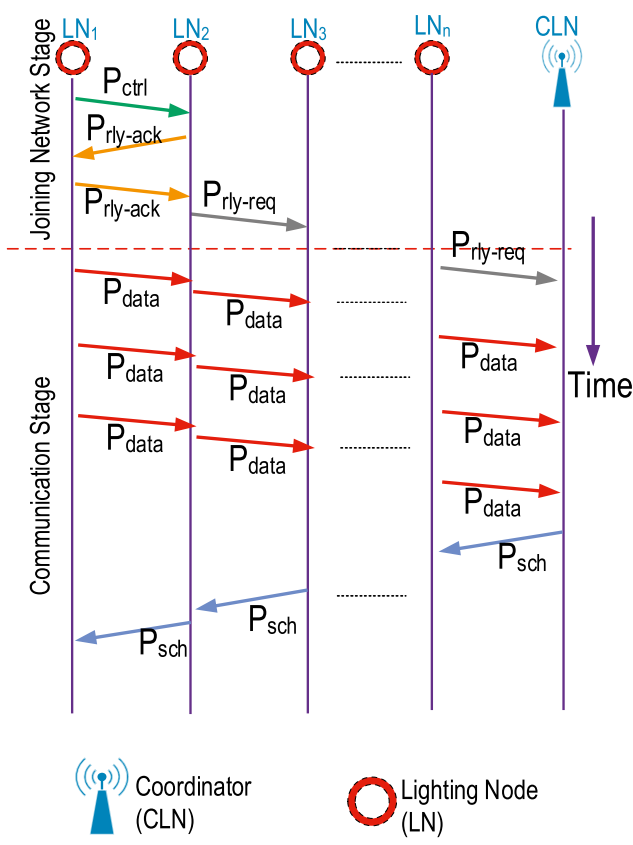

Fig. 4. Proxy-based network joining method

\subsection{Proxy-Based Network Joining}

In multi-hop WSNs, packets of the sensor nodes are transmitted over other nodes and delivered to the coordinator node. Nodes between the target and the source perform their sensing tasks and forward data from other nodes to the next node. Fig. 3 shows the classic approach to network joining.

WSNs adopt a central approach to channel and time slot allocation in classic network scenarios. As shown in Fig. 3, it gets dark in the evening, thus the luminaire sends a request to join the network $\left(P_{c t r l}\right)$ when the power is turned on for the first time. The neighbouring luminaire that receives this request transmits the incoming demand to the next luminaire within its own time slot. The network connection request is then transferred until it reaches the coordinating node (CLN). The coordinator receiving the connection request allocates channels and a time slot for the request. Then it creates a global schedule packet and forwards it along the road that the request came from. The schedule package created by the coordinator is delivered to the end luminaire that makes the request through the luminaires. If the end luminaire finds its own data in the schedule packet, it joins the network by setting its own channel and time slot. After this, the network joining phase is complete. The corresponding luminaire begins transmitting data. With such classic approaches, both time and energy consumption is high. For this reason, organizing the network and 
Table 1. Energy Consumption Values of the Nordic Platform

\begin{tabular}{|c|c|c|c|}
\hline \multirow{2}{*}{$\begin{array}{c}\text { Bandwidth } \\
\text { (KHz) }\end{array}$} & $\begin{array}{c}\text { Data rate } \\
\text { (bps) }\end{array}$ & $\begin{array}{c}\text { Power } \\
\text { consumption } \\
\text { type }\end{array}$ & Power (W) \\
\hline \multirow{2}{*}{100} & \multirow{2}{*}{$5.95 \mathrm{E}+05$} & $P_{T x}$ & 0.0330 \\
\cline { 3 - 4 } 200 & \multirow{2}{*}{$1.19 \mathrm{E}+06$} & $P_{R x}$ & 0.0366 \\
\cline { 3 - 4 } & $P_{T x}$ & 0.0900 \\
\hline \multirow{2}{*}{ No signal } & $P_{R x}$ & 0.0384 \\
\cline { 3 - 4 } & $P_{S p}$ & 0.0003 \\
\hline \multirow{2}{*}{2} & $P_{L P L}$ & $3,75 \mathrm{E}-05$ \\
\hline
\end{tabular}

reconnecting nodes that are disconnected from the network are costly and slow in terms of energy consumption [8, 27].

This study adopted a proxy-based network joining approach in order to improve the energy consumption and performance of the classic approach. Fig. 4 displays a proxy-based approach to connecting to the network.

In the proposed proxy-based network joining method, a local approach is adopted instead of a central system for the channel and time slot assigning. In linear topology, nodes use a common communication channel in a sequence [28]. Nodes in the network use this channel as a time-shared system. When the node attempts to join the network, it sends a request to join the network $\left(P_{c t r l}\right)$ to the neighbour node, as shown in Fig. 4 . When the neighbour node connected to the network receives a request to join the network, it shares own channel and time slot information with that node, allowing it to join the network if appropriate. Only the information of the node that attempts to join the network is sent to the CLN. Thus, the node that attempts to join the network can do it without having to receive a scheduled packet $\left(P_{s c h}\right)$. With this method, the time to join the node to the network becomes much shorter. This ensures that the delay in joining the network, which is sourced from end-to-end packet transmission, is reduced. In addition, the request packet $\left(P_{c t r l}\right)$ is not repeated over other nodes, resulting in a great energy savings.

In this study, the total energy consumptions of $n$ pcs of LN and one CLN during sleep mode, LPL (low power listening mode) and package exchange were calculated as follows [8]. The power consumption of other parameters that may cause to power consumption was ignored. The amount of energy required for a node to receive a packet is calculated according to Eq. 1:

$$
P_{R}=\frac{L_{p k t}}{R_{c h}} P_{R x},
$$

where $P_{R}$ is the power consumed for each packet reception, $P_{R x}$ is the power consumed by the receiver, $L_{p k t}$ is the bit length of the packet, and $R_{c h}$ is the data transmission capacity (bps) value of the transmission channel.

The energy calculation that any LN attempting to send data uses in radio transmission to transmit a packet is shown in Eq. 2:

$$
P_{T}=\frac{L_{p k t}}{R_{c h}} P_{T x},
$$

where $P_{T}$ is the power spent to send each package, $P_{T x}$ is the power of the transmitter circuit power, $L_{p k t}$ is the bit length of the packet, and $R_{c h}$ is the data transmission capacity (bps) of the transmission channel.

The total amount of energy $E_{T}$ expended by a network with $\left(N_{C}=n+1\right)$ nodes consisting of $n$ pcs of LN nodes $(n>2)$ and at least one CLN is calculated as shown in Eq. 3:

$$
E_{T}=\sum_{n=1}^{N_{C}}\left\{\left(P_{R} \cdot N_{R p k t}\right)+\left(P_{T} \cdot N_{T p k t}\right)+P_{S M}+P_{L P L}\right\},
$$

where $N_{R p k t}$ is the number of packets received by the nodes, $N_{T p k t}$ is the number of packets transmitted by the node, $P_{S M}$ is the energy the node spends in sleep mode, and $P_{L P L}$ is the amount of energy the node spends in LPL mode.

As can be seen in Fig. 3, the process of joining a node to the network in the classic approach does not end until the timing packet $\left(P_{s c h}\right)$ sent by CLN reaches the node requesting to join the network. If there are at least 2 or more LNs on the network, then the other nodes between the requesting $\mathrm{LN}$ and the CLN perform the transfer task. LNs in transmitting state, they expend the same amount of energy as $\left(P_{R}+P_{T}\right)$ to receive the packet and then transfer it to the next node. Therefore, in the classic approach, the total amount of energy expended for a node to join the network is as in Eq. 4:

$$
P_{\text {classic }}=N_{C} \cdot\left(P_{R}+P_{T}\right)=(n+1) \cdot\left(P_{R}+P_{T}\right),
$$

where $P_{\text {classic }}$ represents the total energy spent by a node to join in the network based on the classic approach. 


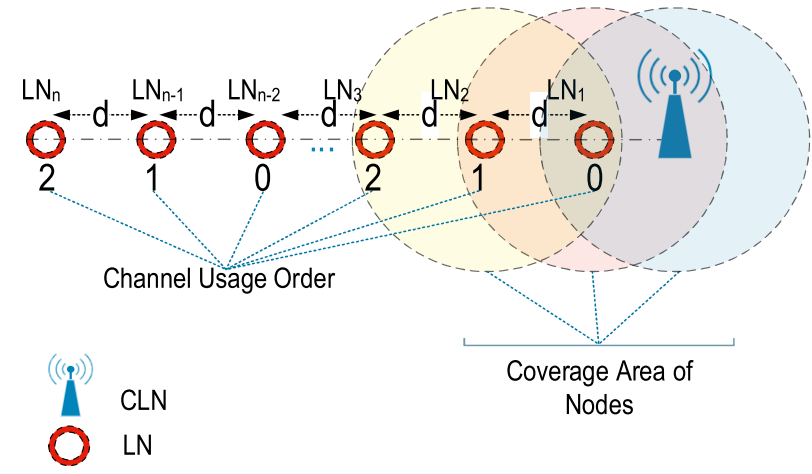

Fig. 5. Schematic representation of the time-division method

Similarly, in the proposed proxy-based network joining approach, as can be seen in Fig. 4, the process of joining a node to the network occurs only by exchanging a packet between the neighbouring node that has joined the network before. At the first moment, there is no need to wait for the schedule packet $\left(P_{s c h}\right)$ sent by CLN. Except of the neighbour $\mathrm{LN}$, other LNs on the network and the CLN do not spend any energy for this process. Therefore, in the proposed proxy-based network joining approach, the total energy spent by a node to join the network is the same as in Eq. 5:

$$
P_{\text {proposed }}=3 \cdot\left(P_{R}+P_{T}\right) \text {. }
$$

In case the control $\left(P_{c t r l}\right)$ and transfer $\left(P_{r l y}\right)$ packet sizes are constant (see Table 2), it is clear that the amount of energy spent by a node to join the network is independent of $N_{C}$ and is constant in the proxy-based network participation approach in Eq. 5. Therefore, the amount of energy $\left(P_{j}\right)$ spent for joining the network by all nodes in a structure with $n$ node is $P_{\text {jclassic }}=n \cdot(n+1) \cdot\left(P_{R}+P_{T}\right)$ in the classic approach, while $P_{\text {jproposed }}=3 \cdot n \cdot\left(P_{R}+P_{T}\right)$ in the recommended proxy-based network joining approach.
Table 2. Types and Sizes of Packaging

\begin{tabular}{|c|c|}
\hline Packet type & Length (byte) \\
\hline Scheduled Packet $($ Psch) & Variable \\
\hline Control Packet (Pctrl) & 32 \\
\hline Relay Packet (Prly) & 52 \\
\hline
\end{tabular}

As a result, as the number of nodes on the network increases, the power spent in the classic approach increases exponentially depending on the number of nodes spent, while the recommended approach will increase linearly. Depending on the fact that the network should have at least two LNs and one CLN, it will always be $P_{\text {jproposed }}<P_{\text {jclassic }}$ $\{n>2\}$.

\subsection{A New Time Division Method}

In classic approaches, collision-based data losses occur because LNs use common channels simultaneously. Therefore, a new time-division method has been proposed to prevent channel access simultaneously with other LNs in the coverage of LNs in linear topology [29].

The proposed time-division method is also implemented in the communication channel that nodes use to transmit data to the CLN. In this method, a node at the $n^{\text {th }}$ level transmits data belonging to itself and its neighbours. If the transmission direction is towards the CLN, it transmits to the node $(n-1)$, and if the transmission direction is downwards from the CLN, it transmits to the node $(n+1)$. In the coverage area of each luminaire, there are two luminaires, the previous one and the next one. A schematic representation of the proposed time-division method is given in Fig. 5.

As shown in Fig. 5, the time-division method is based on how the sensor node uses the channel in a specific sequence order that repeats itself with its neighbour nodes. In this approach, there are two

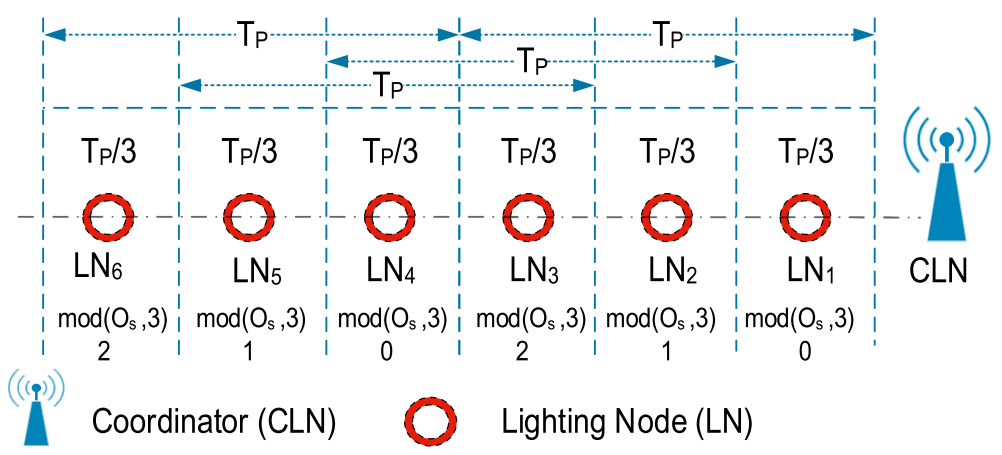

Fig. 6. Time zones for nodes 
Table 3. Simulation Parameters for Scenarios Created for Simulation

\begin{tabular}{|c|c|c|c|c|}
\hline $\begin{array}{c}\text { Scenario/ } \\
\text { Node count }\end{array}$ & Signal coverage of nodes (m) & $\begin{array}{c}\text { Distance between } \\
\text { nodes (m) }\end{array}$ & $\begin{array}{c}\text { Number of } \\
\text { coordinator nodes }\end{array}$ & $\begin{array}{c}\text { Total distance of } \\
\text { network (km) }\end{array}$ \\
\hline S50/50 & 120 & 25 & 1 & 1.25 \\
\hline S100/100 & 120 & 25 & 1 & 2.5 \\
\hline S150/150 & 120 & 25 & 1 & 3.75 \\
\hline S200/200 & 120 & 25 & 1 & 5 \\
\hline
\end{tabular}

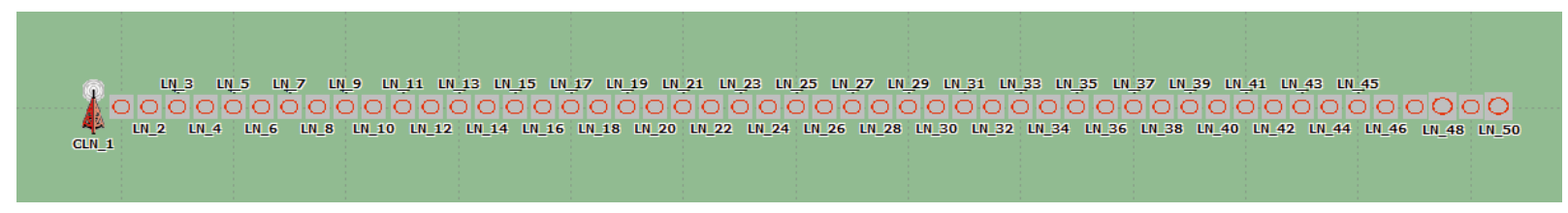

Fig. 7. Linear lighting topology in simulation environment

channels: control channel and data channel. The control channel is used by the luminaire to send the corresponding packets (ctrl, sch, relay) to join the network. In this channel, as the CLN is approached, the data exchange increases due to the property of the linear topology. In the data channel, the LNs transmit data packets to the CLN. Each node transfers both its own data and the data of the lower-level nodes. This channel uses the proposed time division-method. Collision and jam are prevented by using a multi-channel structure in this way. Fig. 6 shows time zones for nodes.

Each node uses the common channel with neighbour nodes in sequence, as shown in Fig. 6. Eq. 7 is used to time division [29]:

$$
S_{d}=\left(\frac{T_{p}}{3}\right) \bmod \left(O_{s}, 3\right),
$$

where $T_{p}$ is the packet transmission period, $S_{d}$ is the node time zone, and $O_{s}$ is the channel allocation order $(0,1,2, \ldots, n)$.

As the LNs join the network, channel usage sequences are determined. The first user is assigned "0". Then $1,2,3, \ldots n$ sequences are assigned to the luminaires in order. LNs learn channel usage sequences with timing packets when they first join the network. When calculating time slots, as seen in Eq. 7, to give nodes an equal time slot, the fixed packet transmission period is division by 3 , and the channel usage order is multiplied by mode (3). As the number of luminaires increases according to the mode of use of the channel (3), it periodically produces results 0,1 and 2 . In this way, each luminaire (LN) on WSN does not use the existing channel simultaneously with the adjacent luminaire through all the way. This prevents collisions and jams. Data is not lost.

\section{SIMULATION AND PERFORMANCE ANALYSIS}

There is no need to pre-determine the locations of the sensor nodes when creating the WSN. In lighting systems, however, the positions of the luminaires are precise and clear. Various WSN protocols have been used in applications for lighting purposes to create a remotely controlled intelligent system [6, 21, 22, 30-33]. The most preferred network systems in these studies are Zigbee and GPRS.

Although using the Zigbee protocol increases energy efficiency, Zigbee has a low data transmission capacity, so it is insufficient when high data transmission is needed. The most commonly used method after Zigbee in remote control applications of lighting is GSM-based GPRS. In many studies in the literature, remote control operation in intelligent lighting systems has been done with GPRSGSM [34-36].

Due to Zigbee's inadequate bandwidth, as well as the high energy consumption of its GPRS-GSMbased WSN protocols, this study preferred $n R F 905$ [37] single chip $433 \mathrm{MHz} / 868 \mathrm{MHz} / 915 \mathrm{MHz}$ transceiver, which is more up-to-date new technologies, easy to use, and has very low energy consumption during data exchange. Thus, nRF905 was used for WSN data exchange in the lighting system.

This research was done for systems with 50, 100, 150 , and 200 road lighting poles with linear topology. A simulation design was preferred for the analysis of the modelling method. The physical implementation of a real application in WSNs results in 


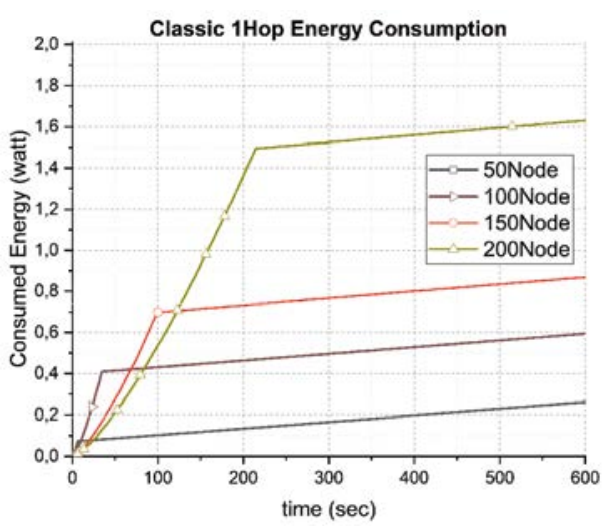

a)

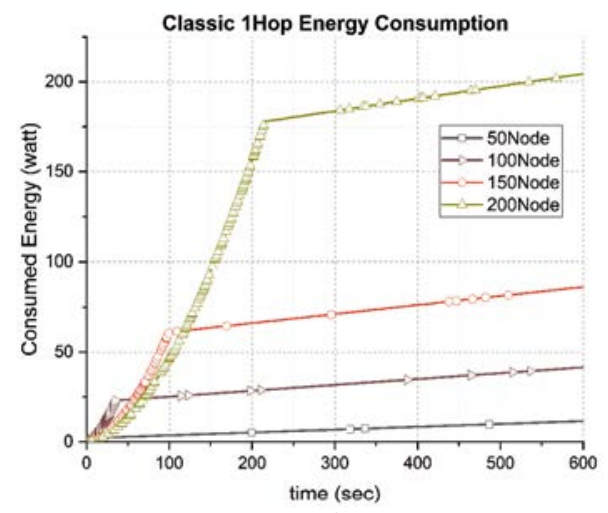

a)

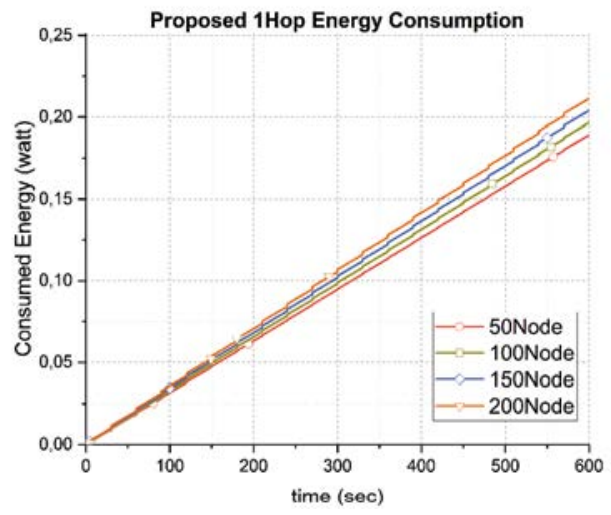

b)

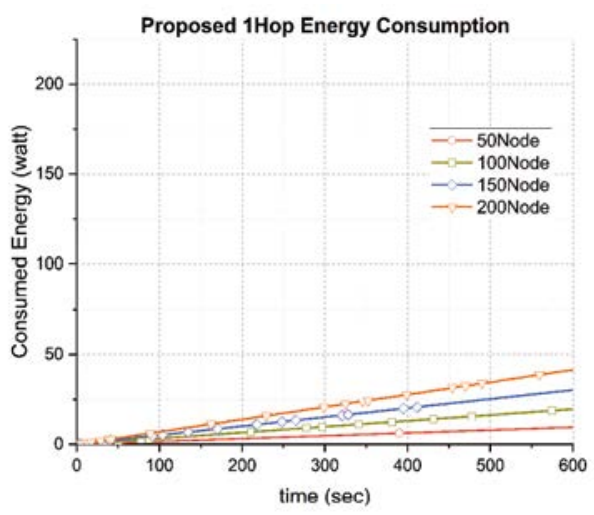

b)
Fig. 8. Comparison of the total energy consumption for single-hop of classical (a) and proposed (b) approaches CLN-based

Fig. 9. The comparison of total LN-based energy consumption for single-hop of classic

(a) and recommended

(b) approaches loss of time, labour and cost. In this case, first, it is necessary to conduct a simulation that produces real-life results.

The developed WSN application was modelled in the Riverbed Modeler simulation design. Riverbed Modeler is a network simulation software where simulation of all network projects can be done [38]. It allows doing many things, such as monitoring the behaviour of the network, performance analysis, testing its superiority. When designing model behaviour, it uses software-specific ProtoC language based on $C$ programming.

Riverbed Modeler enables discrete event-based simulations to analyse the developed network model [10, 23, 38-40].

In this study, two types of nodes were designed in the simulation platform. These nodes are CLN for the central administrative point and LN for luminaires. The topological distribution of the lighting system is designed with linear topology representing road and tunnel lighting. Linear lighting topology in simulation environment is presented in Fig. 7.

The energy consumption of Nordic radios used in node models designed in the simulation environment is presented in Table $1[8,37]$.
One of the parameters that affect energy consumption is the size of data exchange packages used in WSNs. Thus, the WSNs developed as part of this study use 3 different package types for network connectivity and network continuity. Types and sizes of packaging can be seen in Table 2 . The sizes of data packets vary depending on the data to be sent.

The scheduled packet was used by CLN to transmit the channel information assigned to the LNs. Control packets are used by LNs to join directly in the network and maintain continuity in the network. LNs also use relay packets to join the network through other nodes.

In this study, four different scenarios have been developed, and the simulation parameters are shown in Table 3.

The WSN node (LN) numbers are set to 50, 100, 150, and 200 luminaires, respectively, for simulation scenarios. Thus, the effect of increasing the number of nodes on linear topology is seen.

The coverage of the luminous sensors was set to $120 \mathrm{~m}$ for the formation of a single-hop structure. In this way, a luminaire in the linear network can only communicate with two adjacent luminaires. 


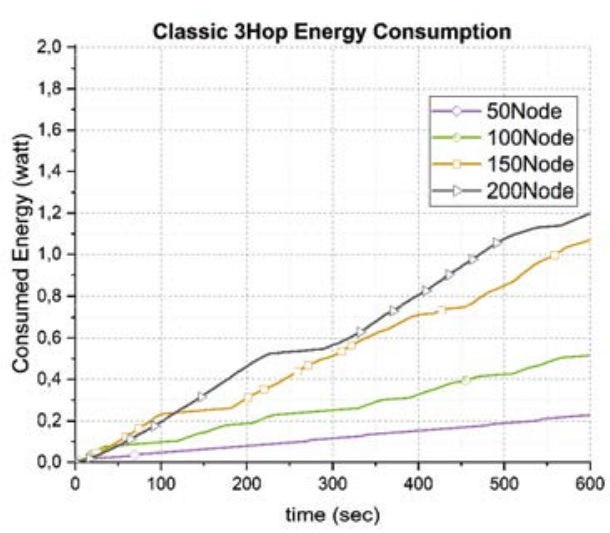

a)

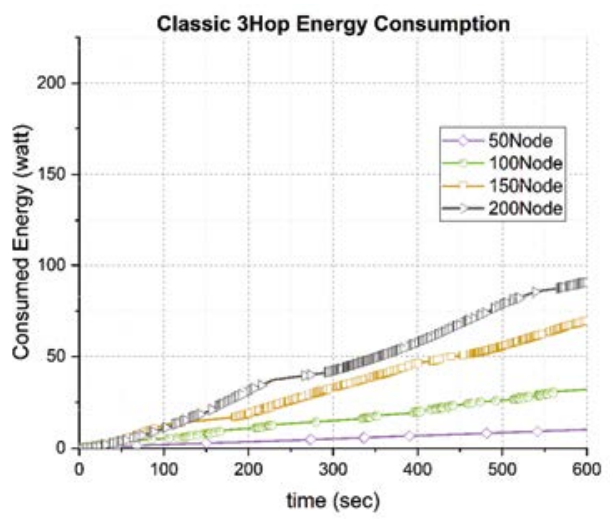

a)

\section{RESULTS AND DISCUSSIONS}

In all scenarios that were simulated in this study, a single CLN was used. Because of simulations, the energy consumption of LNs was examined for single- and multi-hop scenarios. A comparison of networks created using classical approaches and the proposed approaches in this study is made.

First, Fig. 8 shows comparison of the total energy consumption of classical (a) and proposed (b) for single-hop approaches CLN-based.

As shown in Fig. 8, in the classical approach, a sudden energy demand occurs at the moment of joining the network. The energy consumed by the CLN of the 200 luminaires system, which consumes the most energy at the first moment, is $1.5 \mathrm{~W}$ when all LNs join the network. In the proposed approach, the energy consumption of CLN is $0.0116 \mathrm{~W}$ for the same situation. The advantage of all LNs joining the network is $99.23 \%$. This can be explained as follows: in the classic approach, LNs create severe packet traffic to join the network at the first moment. The CLN processes every connection request that reaches it and responds back, thus expending a lot of energy. Since the proposed approach
Proposed 3Hop Energy Consumption

Properion

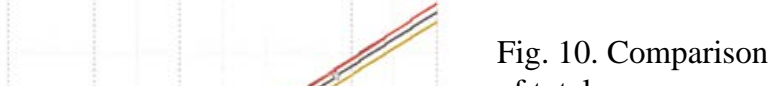
of total energy consumption of the classical (a) and the proposed (b) approaches CLN-based for 3 hops

Fig. 11. Comparing of the total energy consumption of classic (a) and recommended (b) approaches LN-based for 3-hop

adopts a proxy-based network connection, the relevant neighbour LN, not CLN, makes the network connection process. This allows the CLN to be less busy with fewer packets. This reduces the energy expended by the CLN during the network connection phase. For other scenarios, this situation is the same as can be seen in Fig. 8. With a similar approach, LN-based total energy consumption for single-hop is shown in Fig. 9.

As shown in Fig. 9, LNs create heavy packet traffic in the classic approach to join the network at the first moment. In the experiment of $200 \mathrm{LNs}$, which is the most difficult scenario, the energy consumed by the nodes is $175 \mathrm{~W}$, while the energy consumption in the proposed approach is $0.235 \mathrm{~W}$. The advantage provided is $99.86 \%$.

Fig. 10 (a) shows the amount of energy spent by the CLN in simulation scenarios with 50,100 , 150, and 200 lighting poles with 3 hops in the classical approach. In the scenario of $200 \mathrm{LN}$, which is the most difficult scenario in the classical approach, the process of connecting all the LNs to the network takes 225 seconds. At this point, the CLN consumed $0.552 \mathrm{~W}$. In the proposed approach shown in Fig. 10 (b), the power consumption of the CLN is 
Table 4. Total LNs Energy Consumption by Hop Numbers for the Simulations of 200 LNs

\begin{tabular}{|c|c|c|c|}
\hline & 1-hop & 2-hop & 3-hop \\
\hline Classic (W) & 205.92 & 89.71 & 91.81 \\
\hline Proposed (W) & 41.63 & 37.59 & 38.57 \\
\hline Advantage (\%) & $\sim 80$ & $\sim 58$ & $\sim 58$ \\
\hline
\end{tabular}

Table 5. Total CLN Energy Consumption Based on Hop Numbers for Simulation of 200 LNs

\begin{tabular}{|c|c|c|c|}
\hline & 1-hop & 2-hop & 3-hop \\
\hline Classic (W) & 1.63 & 1.08 & 1.20 \\
\hline Proposed (W) & 0.211 & 0.200 & 0.205 \\
\hline Advantage (\%) & $\sim 87$ & $\sim 81$ & $\sim 83$ \\
\hline
\end{tabular}

Table 6. Total (CLN + 200 LNs) Energy Consumption of the System Based on Hop Numbers for Simulation of $200 \mathrm{LNs}$

\begin{tabular}{|c|c|c|c|}
\hline & 1-hop & 2-hop & 3-hop \\
\hline Classic (W) & 207.55 & 90.79 & 93.010 \\
\hline Proposed (W) & 41.841 & 37.79 & 38.775 \\
\hline Advantage (\%) & $\sim 80$ & $\sim 58$ & $\sim 58$ \\
\hline
\end{tabular}

only $0.0086 \mathrm{~W}$ in the most difficult scenario of 200 LNs, when all the LNs join to the network. Accordingly, the proposed approach provides an advantage of $98.35 \%$ in the network connection phase of LNs compared to the classical approach.

Similarly, Fig. 11 shows comparison of the total energy consumption of the classic (a) and proposed (b) the LN-based approaches for 3-hop.

According to Fig. 11, in the analysis of 200 LN that is the most difficult scenario of the classic method with 3-hop, the energy consumption required for all LNs to join the network in full performance is $39 \mathrm{~W}$. In the proposed approach of this study, the energy consumed is $0.387 \mathrm{~W}$ for the same scenario. Here, the proposed approach provides a $99 \%$ advantage.

According to the node numbers (LN) of the network, the comparison of the total energy consumption of LNs in the simulation scenarios 50, 100,150 , and $200 \mathrm{LNs}$ for different hop numbers of the classical and proposed approaches is shown in Fig. 12.

In Fig. 12, the total amounts of energy expended by all LNs (except CLN) in connection between LNs in 1-hop, 2-hop, and 3-hop scenarios were compared in terms of the classical and proposed method. In this study, it was observed that the clas-

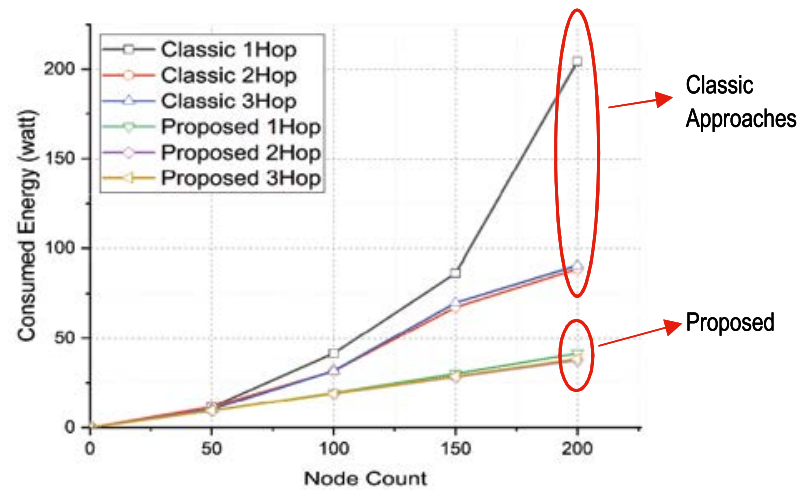

Fig. 12. Comparison of the total energy consumption of LNs

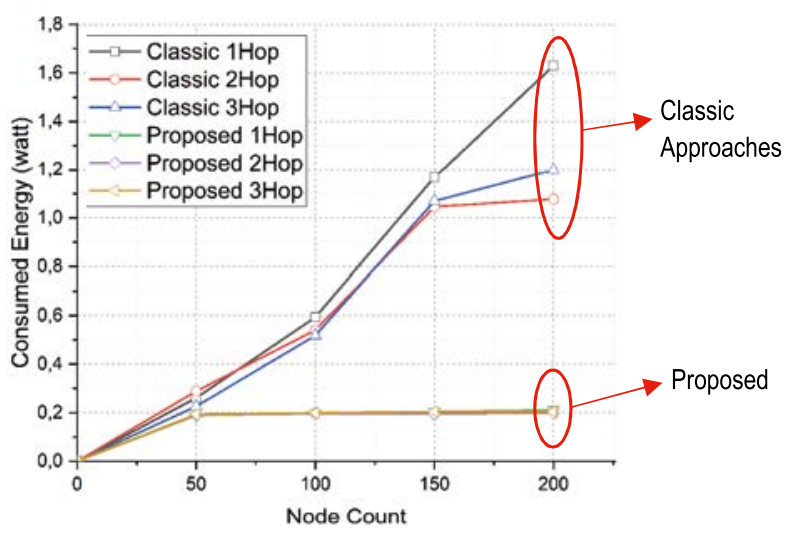

Fig. 13. CLN energy consumption comparison

sic method consumes much more energy. Accordingly, the total energy consumption and savings obtained according to the hop numbers for the 200 LNs simulation that is the most difficult scenario are seen in Table 4.

The comparison of CLN energy consumption in 50, 100, 150, and 200 LNs simulation scenarios for different hop numbers of classic and recommended approaches is shown in Fig. 13.

As shown in Fig. 13, the energy that CLN expends in the 1-hop, 2-hops and 3-hops is compared in terms of the classical and proposed method. In all scenarios of 50,100, 150, and $200 \mathrm{LNs}$, it is seen that the proposed method is much more efficient in terms of total energy consumption. For example, in a linear topology scenario with 200 poles, the comparison of energy consumption and the resulting savings amounts are shown in Table 5.

Table 6 shows the total (CLN + 200 LNs) energy consumption of the system based on hop numbers for simulation of 200 LNs.

Table 6 shows the total energy consumption of the system with 200 luminaires, which is the most difficult scenario. When comparing the classic and 
proposed approaches, you can see savings of $\sim 80 \%$, especially in a single hop situation.

\section{CONCLUSION}

In the classic approach, the energy consumption during the first join of the LNs to the network is higher, and this is due to the frequent use of schedule, control and relay packets at the first time the system is energized. In the proposed approach, the proxy-based network joining method eliminates this situation. The proposed approach gives an advantage of $98.23 \%$ in a single-hop structure. Thus, the minimum energy consumption is achieved during the connection phase to the network, where the energy is consumed the most.

The excess of total energy consumption is particularly prominent in linear scenarios. The main reason for the increase in total energy consumption is related to the increase in the number of luminaires. As the number of LNs increases, the maximum number of hops in the system will also increase. Each hop means extra packet transfer and repetition. This will result in more energy consumption.

The approach to implementing WSN based on linear topology proposed in this study works better than the classic approach. As the number of LNs increased, the packet traffic of the LNs close to the CLN increased, but no collision was seen. End-toend delay, one of the most important performance parameters in linear topologies, is acceptable, although the number of LNs increases. All scenarios in this study used a single CLN and were intended to maximize WSN's performance under these circumstances. On the other hand, as the number of CLN increased, it was observed that LNs connected to the network faster and that the end-to-end delay was much less. In this study, all LNs in WSN used the recommended time-division method for data transmission and control communication.

In simulations, it can be observed that as the number of LNs increases in the classical approach, the length of time that nodes will be involved in the network increases. Also, in the same scenarios with the numbers of nodes $50,100,150$, and 200 , the time for joining nodes to the network increases parabolically (5 sec., 18 sec., 99 sec., 210 sec., respectively). In WSN applications, where the number of nodes is much bigger, these periods are much longer. However, due to the proxy-based network join- ing method proposed in this study, it could be observed that these times are very much decreased to very low acceptable values $(0.3 \mathrm{sec}$., 0.89 sec., $1.53 \mathrm{sec}$., and $2.4 \mathrm{sec}$.). In addition, the proposed approaches enabled quicker organization of the network. In case of a possible breakaway, the network the network itself will quickly reorganize.

As a result, the development of energy- and time-sensitive methods in linear WSN applications used in lighting systems is imperative in terms of network performance and efficiency of the application. Thanks to the proposed solutions, energy consumption was reduced by $80 \%$ and $58 \%$ depending on different scenarios.

\section{REFERENCES}

1. Dong P. Application of intelligent lighting control system in different sports events in sports venues. Light \& Engineering, 2018. V26, \#4, pp. 165-171.

2. Cheng R. Classroom Lighting Energy-Saving Control System Based on Machine Vision Technology. Light \& Engineering, 2018. V26, \#4, pp. 143-149.

3. Sun E. Solar Photovoltaic Power Generation Wireless Monitoring System Based on IOT Technology. Light \& Engineering, 2018. V26, \#4, pp. 130-136.

4. Sanaz Bozorg Chenani, Rami-Samuli Rasanen, Eino Tetri, Advancement in Road Lighting. Light \& Engineering, 2018. V26, \#2, pp. 99-109.

5. Iacomussi P., Rossi G., Soardo P. Energy saving and environmental compatibility in road lighting. Light \& Engineering, 2012. V20, \#4, pp. 55-63.

6. Hao L., Gao J. Intelligent Lighting System of Urban Road Based on Internet of Things. Light \& Engineering, 2018 V26, \#4, pp. 150-156.

7. Cao S. Intelligent Lighting Control System in Large-Scale Sports Competition Venues. Light \& Engineering, 2018. V26, \#4, pp. 172-182.

8. Çıbuk M., Cengiz M.S. Determination of Energy Consumption According To Wireless Network Topologies In Grid-Free Lighting Systems. Light \& Engineering, 2020. V28, \#2, pp. 67-76.

9. Zou Q. Lighting and Control Design of LargeScale Stadium Skating Competition. Light \& Engineering, 2018. V26, \#4, pp. 183-190.

10. Arı D., Çıbuk M., Ağgün F. Effect of Relay-Priority Mechanism on Multi-hop Wireless Sensor Networks. Bitlis Eren University Journal of Science and Technology, 2017. V7, \#2, pp. 145-153.

11. Çıbuk M. A New Fast Network Joining Algorithm for Single-Hop Wireless Sensor Networks. Bitlis 
Eren Üniversitesi Fen Bilimleri Dergisi, 2018. V7, \#1, pp. 72-83.

12. Shrestha A., Xing L. A performance comparison of different topologies for wireless sensor networks, in 2007 IEEE Conference on Technologies for Homeland Security: Enhancing Critical Infrastructure Dependability, 2007. pp. 280-285.

13. Mamun Q. A Qualitative Comparison of Different Logical Topologies for Wireless Sensor Networks. Sensors, 2012. V12, \#11, pp. 14887-14913.

14. Efe S. B. UPFC Based Real-Time Optimization of Power Systems for Dynamic Voltage Regulation. Computer Modeling in Engineering \& Sciences, 2018. V116, \#3, pp. 391-406.

15. Efe S. B., Cebeci M. Power Flow Analysis by Artificial Neural Network. International Journal of Energy and Power Engineering, 2013. V2, \#6, pp. 204-208.

16. Cengiz M.S. Simulation And Design Study For Interior Zone Luminance In Tunnel Lighting. Light \& Engineering, 2019. V27, \#2, pp. 42-51.

17. Yetgin H., Cheung K.T.K. El-Hajjar, M., Hanzo, L., A Survey of Network Lifetime Maximization Techniques in Wireless Sensor Networks. IEEE Communications Surveys \& Tutorials, 2017. V19, \#2, pp. 828-854.

18. Cengiz M. S., Cengiz Ç. Numerical Analysis of Maintanance Factor for Tunnel and Road In Solid State Lighting, in International GAP Renewable Energy and Energy Efficiency Congress, 2018. pp. 347-348.

19. Cengiz M. S., Cengiz Ç. Numerical Analysis of Tunnel LED Lighting Maintenance Factor. IIUM Engineering Journal, 2018. V19, \#2, pp. 154-163.

20. Cengiz M.S. The Relationship between Maintenance Factor and Lighting Level in Tunnel Lighting. Light \& Engineering, 2019. V27, \#3, pp. 75-88.

21. Peixoto J. P. J., Costa D.G. Wireless visual sensor networks for smart city applications: A relevance-based approach for multiple sinks mobility. Future Generation Computer Systems, 2017. V76, pp. 51-62.

22. Karun R., Johny M. Street Light Commander System Using Zigbee Network of Devices. International Journal of Engineering and Innovative Technology (IJEIT), 2014. V4, \#4, pp. 165-169.

23. Çıbuk M., Arı D., Ağgün F., Relay Mechanism with Three-way Handshake for Wireless Sensor Networks, in International Advanced Technologies Symposium (IATS17), 2017. pp. 3459-3466.

24. Bathla M., Sharma N. Topology Control in Wireless Sensor Networks. International Journal of Advances in Computer Networks and its Security, 2009. pp. 161-164.
25. Varshney S., Kumar C., Swaroop A. Linear Sensor Networks: Applications, Issues and Major Research Trends, in International Conference on Computing, Communication and Automation (ICCCA2015), 2015. pp. 446-451.

26. Chen Q., Wang K., Ying K., Miao C., Dai G. An Energy Efficient MAC Protocol for Linear WSNs. Chinese Journal of Electronics, 2015. V24, \#4, pp. 725-728.

27. Çıbuk M., Arı D., Çınar H. A New Multi-Channel Algorithm Of Join The Network For Single Hop MultiNode Sensor Networks, in International Engineering, Science and Education Conference - INESEC2016, 2016. \#December, pp. 1-3.

28. Arı D., Çıbuk M., Ağgün F. A New Proxy-Based Network Joining Method for Linear Wireless Sensor Networks, in International Engineering and Natural Sciences Conference (IENSC2018), 2018. pp. 715-723.

29. Arı D., Çıbuk M., Ağgün F. Doğrusal Kablosuz Algılayıcı Ağlar için Yeni Bir Zaman Dilimleme Metodu, in $1^{\text {st }}$ International Engineering and Technology Symposium, 2018. pp. 1166-1170.

30. Srinath V., Srinivas S. Street Light Automation Controller using Zigbee Network and Sensor with Accident Alert System. International Journal of Current Engineering and Technology, 2015. V5, \#4, pp. 2819-2823.

31. Bhargavi R., Busupalli P. Development of Automatic Street Light Illumination and Vehicle Speed Controlling System on Arm7 for Roadways. International Journal of Research in Advanced Engineering Technologies, 2016. V5, \#3, pp. 16-22.

32. Gomez C., Paradells J. Wireless Home Automation Networks- A Survey of Architectures and Technologies.pdf. CONSUMER COMMUNICATIONS AND NETWORKING, 2010. \#June, pp. 92-101.

33. Radmand P., Talevski A., Petersen S., Carlsen S. Comparison of industrial WSN standards. 4th IEEE International Conference on Digital Ecosystems and Technologies - Conference Proceedings of IEEE-DEST 2010, DEST 2010, 2010. pp. 632-637.

34. Caponetto R., Dongola G., Fortuna L., Riscica N., Zufacchi D. Power consumption reduction in a remote controlled street lighting system, in SPEEDAM 2008 International Symposium on Power Electronics, Electrical Drives, Automation and Motion, 2008. pp. 428-433.

35. Dingfang L., Suiping Q., Tundong L., Shou-Zhi Y., Funchun S. The design and realization of communication technology for street lamps control system. Proceedings of 2009 4th International Conference on Computer Science and Education, ICCSE2009, 2009. pp. 259-262.

36. Nasirudin M. A., Za'bah U. N., Sidek O. Fresh water real-time monitoring system based on Wireless Sen- 
sor Network and GSM. Open Systems (ICOS), 2011 IEEE Conference on, 2011. pp. 354-357.

37. Nordic Semiconductor, nRF905 Single chip 433/868/915MHz Transceiver, 2008.

38. Riverbed, OPNET Technologies - Network Simulator, 2018. https://www.riverbed.com/gb/products/steelcentral/opnet.html (accessed May 02, 2018).

39. Vançin S., Erdem E. Design and simulation of advance MODLEACH for wireless sensor network, in Inter- national Journal of Computer Networks and Applications (IJCNA), 2015. V2, \#3, pp. 135-143.

40. Cengiz M. S., Cengiz Ç. IOT and Lighting Automation, International Conference on Multidisciplinary, Engineering, Science, Education and Technology (IMESET’17 Baku) Book of Abstracts, 12-14 July 2017, Baku, pp. 8.

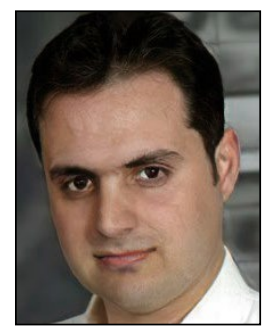

Musa Cıbuk,

received his M. Sc. and Ph.D. degrees from Frrat University, Turkey in 2002 and 2009, respectively. His research interests include WSNs, MAC, Computer Networks, Digital Communication and Image Processing. From 2000 to 2010, he worked at the University of Firat. Currently, he works at Bitlis Eren University, serving as the Head of Department at Computer Engineering 